\title{
Erratum to: Strategies of laparoscopic thyroidectomy for treatment of substernal goiter via areola approach
}

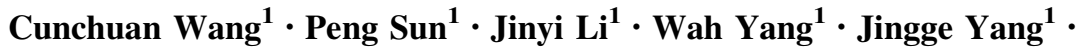
Zhiqi Feng ${ }^{1} \cdot$ Guo Cao $^{1} \cdot$ Shing Lee ${ }^{1}$

Published online: 7 July 2016

(C) Springer Science+Business Media New York 2016

\section{Erratum to: Surg Endosc}

DOI 10.1007/s00464-016-4814-0

Figures 2, 3, and 4 were submitted in error. Revised Figs. 2, 3, and 4 are now included.

The online version of the original article can be found under doi:10.1007/s00464-016-4814-0.

\section{Cunchuan Wang}

wangcunchuan@tom.com; twcc@jnu.edu.cn

1 Department of General Surgery, First Affiliated Hospital of Jinan University, Guangzhou 510630, China 


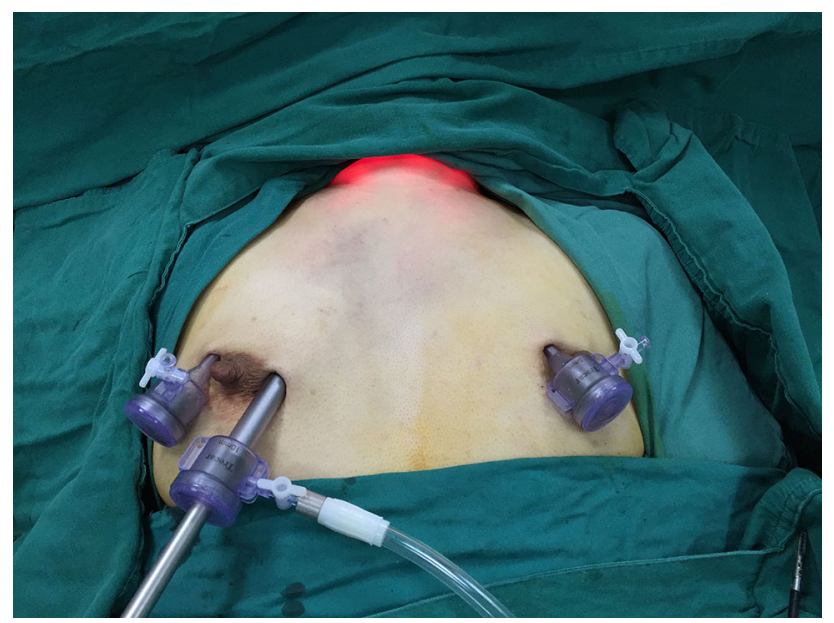

Fig. 2 External view after positioning the trocars

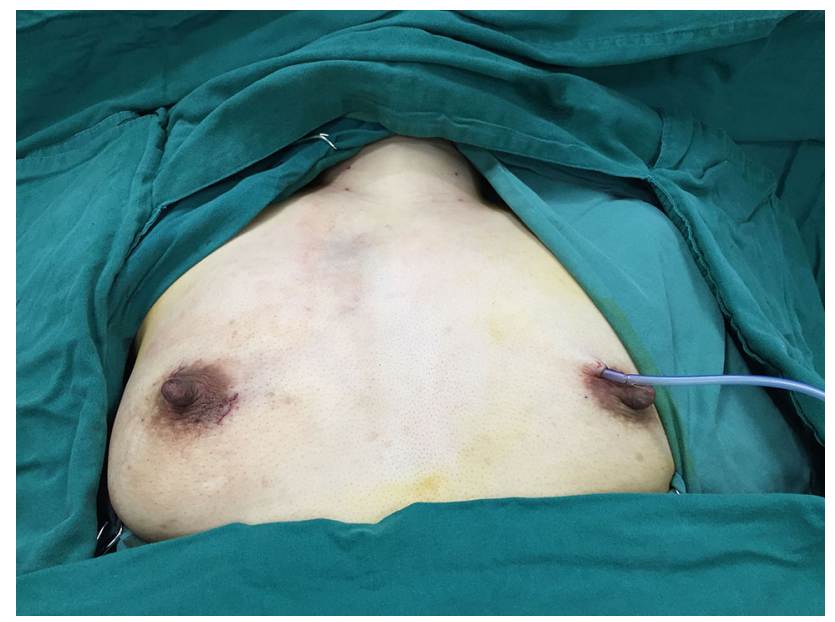

Fig. 3 Incisions of endoscopic thyroidectomy for treatment of substernal goiter via areola approach

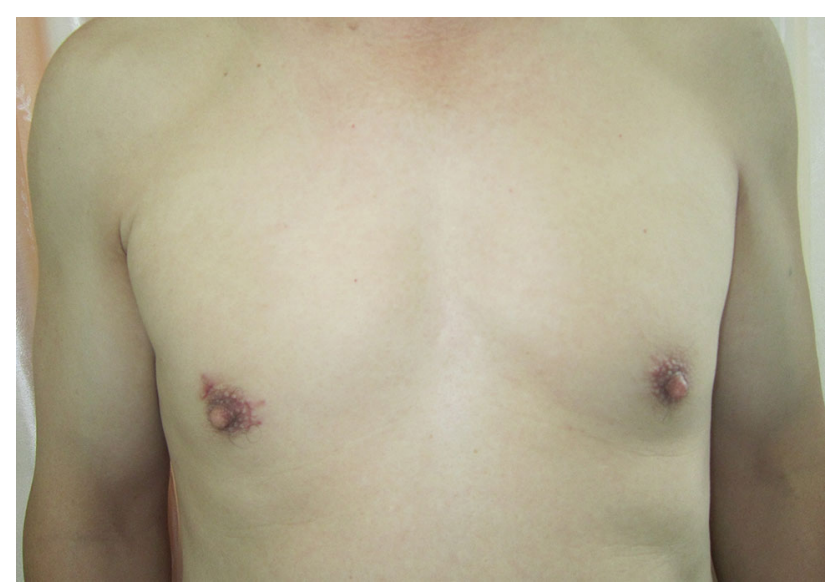

Fig. 4 Incision scars are unconspicuous and concealed (3 months after surgery) 